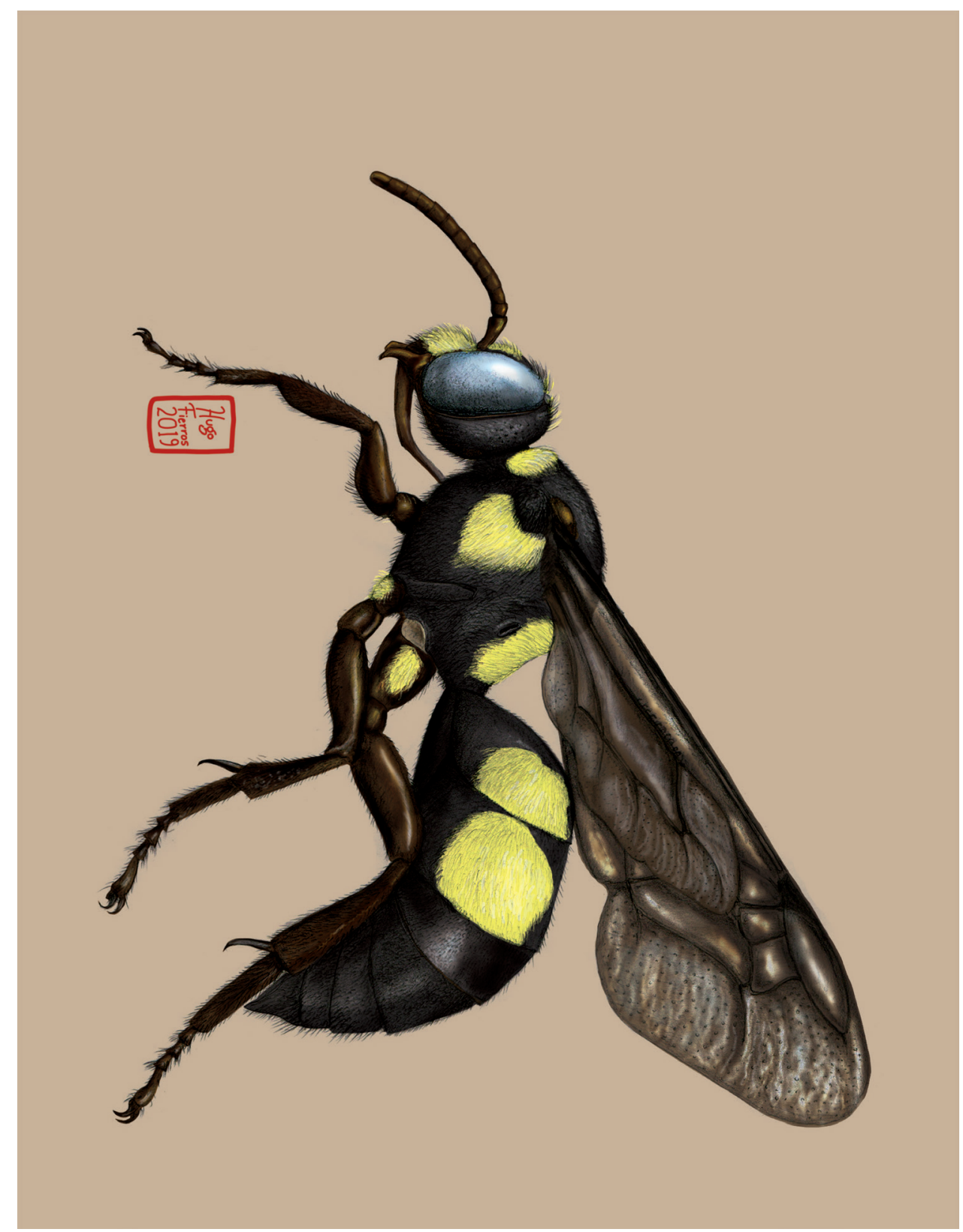

Dugesiana, Año 27, No. 1, enero 2020-junio 2020 (primer semestre de 2020), es una publicación semestral, editada por la Universidad de Guadalajara, a través del Centro de Estudios en Zoología, por el Centro Universitario de Ciencias Biológicas y Agropecuarias. Camino Ramón Padilla Sánchez \# 2100, Nextipac, Zapopan, Jalisco, Tel. 37771150 ext. 33218, http://148.202.248.171/dugesiana/index.php/DUG/index, glenusmx@gmail.com. Editor responsable: José Luis Navarrete-Heredia. Reserva de Derechos al Uso Exclusivo 04-2009-062310115100-203, ISSN: 2007-9133, otorgados por el Instituto Nacional del Derecho de Autor. Responsable de la última actualización de este número: José Luis Navarrete-Heredia, Editor y Ana Laura González-Hernández, Asistente Editorial. Fecha de la última modificación 1 de enero 2020, con un tiraje de un ejemplar.

Las opiniones expresadas por los autores no necesariamente reflejan la postura del editor de la publicación.

Queda estrictamente prohibida la reproducción total o parcial de los contenidos e imágenes de la publicación sin previa autorización de la Universidad de Guadalajara. 


\title{
Diversidad de hormigas (Hymenoptera: Formicidae) de La Cantera Oriente, una reserva den- tro de la ciudad de México
}

\author{
Diversity of ants (Hymenoptera: Formicidae) of La Cantera Oriente, a reserve in Mexico city
}

\author{
Paola García Villar ${ }^{1}$ y Leticia Ríos-Casanova ${ }^{2}$ \\ Laboratorio de Ecología, Unidad de Biotecnología y Prototipos, Facultad de Estudios Superiores Izta- \\ cala, Universidad Nacional Autónoma de México. Av. de los Barrios 1, Los Reyes Iztacala, Tlalnepantla \\ 54090, Estado de México, México. 'gaara_uchan@hotmail.com ; ${ }^{2}$ leticiarc@unam.mx
}

\begin{abstract}
RESUMEN
Se estudió la diversidad de hormigas de La Cantera Oriente, una zona restaurada de la Reserva Ecológica del Pedregal de San Ángel en la Ciudad de México con el objetivo de conocer dicha diversidad en dos zonas y durante la temporada de lluvia y sequía, además de comparar esta diversidad con la de una zona conservada de la misma reserva. Para llevarlo a cabo, se midió la riqueza y abundancia de las hormigas en cada zona y temporada, además correlacionamos las medidas de diversidad con la estructura de la superficie del suelo y con la estructura vertical de la vegetación. Finalmente calculamos el índice de Sorensen para comparar la riqueza de La Cantera y de la de una zona conservada de la reserva. Encontramos diez especies de hormigas, las especies más abundantes fueron Monomorium minimum Buckley, 1866 y Paratrechina longicornis Latreille, 1802. Aunque en la temporada seca se presentó mayor abundancia, riqueza y diversidad, no se encontraron diferencias significativas entre sitios o temporadas para estas variables. Únicamente la abundancia se correlacionó con la proporción de pasto durante la temporada de lluvia. Siete especies de La Cantera Oriente se comparten con las de una zona conservada de la reserva. Concluimos que la diversidad de hormigas de La Cantera Oriente no es afectada por los cambios espaciales y temporales de este lugar. La similitud entre las especies de la zona conservada de la Reserva y las de La Cantera es muy baja, lo que podría asociarse al alto grado de perturbación que tuvo esta última.

Palabras clave: comunidades de hormigas, estructura de la superficie del suelo, hormigas invasoras, Monomorium minimum, Paratrechina longicornis, restauración.

ABSTRACT

In this work we study the ant diversity of La Cantera Oriente, a recently restored area that is part of the Ecological Reserve El Pedregal de San Ángel in Mexico City. Our main issue was to know the diversity of ants in two areas and during the rainy season and drought. In addition, we compare this diversity with that of a conserved area of the same reserve. In order to do this, we measure species richness and abundance of ants in two different sites of La Cantera Oriente during the rainy and drought seasons. We also correlated diversity measures with the structure of the soil surface and with the vertical structure of the vegetation. Finally, we calculated the Sorensen index to compare species richness of La Cantera and a conserved zone of the reserve. We found ten species of ants of the subfamilies Dolichoderinae, Formicinae and Myrmicinae. The most abundant species were Monomorium minimum Buckley, 1866 and Paratrechina longicornis Latreille, 1802. Although during the drought there is greater abundance, richness and diversity of ants, we did not find significant differences between sites or seasons for these variables. We also found that only abundance was correlated with the proportion of grass during the rainy season. Seven species of La Cantera Oriente are shared with those of the conserved area of the reserve. We conclude that ant diversity of La Cantera Oriente is not affected by the spatial and temporal changes of this place. The similarity between the species of the core zone of the Reserve and those of La Cantera is very low, which could be associated with the high degree of disturbance that the latter had.
\end{abstract}

Key words: ant communities, invasive ants, Monomorium minimum, Paratrechina longicornis, restoration, soil surface structure.

Las hormigas son un grupo de insectos con gran diversidad funcional y taxonómica (Höldobler y Wilson 1990). En diferentes ecosistemas se desempeñan como depredadoras, herbívoras o detritívoras, participan en la descomposición y reciclaje de nutrientes del suelo y son dispersoras de semillas (Folgarait 1998). Existe evidencia que sugiere que la diversidad está positivamente relacionada con la complejidad del hábitat ya que ésta crea una mayor disponibilidad de nichos: entre más complejo sea un sistema, mayor es la pro- babilidad de que los recursos sean divididos y así mismo, la riqueza de la flora y la fauna sea mayor (Retana y Cerdá 2000; Tews et al. 2004). Sin embargo, la urbanización es un proceso en el que se pierden los espacios naturales y se modifican las condiciones del ambiente con la consecuente disminución de la diversidad de plantas y de la cobertura vegetal convirtiendo al ambiente en un sistema más simple y homogéneo (Clarke et al. 2008). Dichas variaciones pueden propiciar cambios en la composición y dinámica de las 
comunidades de hormigas que se desarrollan en sitios urbanizados o en proceso de urbanización (Clarke et al. 2008). Sin embargo son muy pocos los estudios que analizan las variables ambientales que explican la estructura, composición y riqueza de las especies de hormigas urbanas (Santos, 2016).

En los estudios realizados en zonas urbanas se sugiere que entre los principales factores que determinan la abundancia y distribución de las hormigas se encuentran la simplificación de la estructura de la vegetación y del suelo, la compactación del suelo y las altas temperaturas, pues estos son factores que generalmente disminuyen la diversidad (Clarke et al. 2008).

Otro factor importante es el origen de la vegetación, ya que los parches con vegetación nativa dentro de zonas urbanas tienen mayor número de especies de hormigas (Pacheco y Vasconcelos 2007). La Ciudad de México es una de las zonas del país con mayor población humana y mayor densidad, por lo que presenta problemas ambientales severos (Chávez y Ceballos 1998). Sin embargo, existen áreas naturales protegidas dentro de la ciudad como la Reserva Ecológica del Pedregal de San Ángel (REPSA), la cual representa un lugar dentro de la Ciudad de México que aún conserva una gran diversidad biológica (Lot y Cano-Santana 2009). Dicha diversidad es resultado de diversos factores como su origen geológico y su topografía accidentada, lo que ha originado un ecosistema con una composición biótica particular (Álvarez-Sánchez et al. 1982).

La REPSA está actualmente dividida en zonas núcleo y zonas de amortiguamiento. Una de estas zonas de amortiguamiento es La Cantera Oriente la cual es particularmente interesante ya que fue utilizada para la extracción de material basáltico durante 25 años (de 1970 a 1994) y en la que hace 21 años se introdujeron plantas exóticas y suelo con el fin de restaurarla (Lot 2007).

Actualmente La Cantera Oriente tiene sitios en los que predominan árboles y pastos introducidos y otros en los que además de las plantas introducidas aun crecen algunas plantas típicas del Pedregal y que son las que se desarrollan con mayor abundancia en las zonas núcleo que están mejor conservadas. Por lo anterior, en este trabajo se estudió la diversidad de hormigas de dos sitios contrastantes de La Cantera Oriente, uno muy transformado durante la restauración y otro también muy transformado pero que conserva algunas de las plantas típicas de la reserva. Finalmente se comparó la riqueza de especies de hormigas de la Cantera Oriente con la de una zona núcleo de la reserva (más conservada) esperando que esta última presentará la mayor riqueza específica de hormigas.

\section{MATERIAL Y MÉTODOS}

Área de estudio. La Cantera Oriente de la REPSA está localizada al sur de la Ciudad de México (19 32' 02 'N - $99^{\circ} 17^{\prime} 37^{\prime}$ W). Tiene un clima templado sub-húmedo con una precipitación promedio anual de $833 \mathrm{~mm}$ y una temperatura media anual de $15.6^{\circ} \mathrm{C}$. Presenta una marca- da estacionalidad que comprende una época de lluvias de junio a octubre y una de sequía de noviembre a mayo (Lot 2007). Actualmente es una de las zonas de amortiguamiento pertenecientes a la REPSA (zonas A3), presenta sitios fisonómicamente distintos, por lo que en este estudio se consideraron el sitio Jardín y el sitio Sendero (Lot 2007 y observaciones personales). El sitio Sendero corresponde al camino principal de La Cantera, es uno de los sitios más heterogéneos ya que de un lado presenta cipreses (Cupressus sp.) y del otro vegetación típica de la REPSA como Echeverria gibiflora D.C., el árbol Budleia cordata (Kunth) y el arbusto Verbesina virgata Cav. El Jardín es un sitio creado con propósitos ornamentales, presenta árboles como cipreses y fresnos (Fraxinus sp.) y gran cobertura de pasto (Pennisetum clandestinum Hochst) (Lot et al. 2012; Fig.1).

Diversidad de hormigas. Se realizó la colecta de hormigas en cuatro ocasiones, dos en época de sequía (enero y marzo de 2014) y dos en lluvia (agosto y octubre 2014). Durante cada colecta, en cada sitio se colocaron 10 trampas de caída, cada $10 \mathrm{~m}$ en dos transectos de $40 \mathrm{~m}$ de longitud, separados $10 \mathrm{~m}$ entre sí. Las trampas consistieron en recipientes de plástico de $60 \mathrm{~mm}$ de diámetro y $100 \mathrm{~mm}$ de altura conteniendo $100 \mathrm{ml}$ de una mezcla de $70 \%$ de etanol $\left(96^{\circ}\right)$ y $30 \%$ de agua. Las trampas se enterraron a ras de suelo, las cuales permanecieron cerradas por $24 \mathrm{~h}$ para minimizar el efecto de la excavación, posteriormente se mantuvieron destapadas por $48 \mathrm{~h}$, al término de las cuales se cerraron y transportaron al laboratorio donde se determinaron a nivel de género usando la clave de Fisher y Cover (2007). La mayoría de las hormigas se determinaron a nivel de especie usando claves para cada género y con la colaboración del Dr. Miguel Vásquez Bolaños (Universidad de Guadalajara, México) quién realizó y/o corroboró las determinaciones.

Debido a que el análisis de la diversidad puede afectarse fuertemente por el esfuerzo de colecta, se construyeron curvas de acumulación de especies, observadas y esperadas, para cada sitio y temporada. Para obtener la curva de especies esperadas, se utilizó el programa EstimatesS 8.2.0 realizando 100 aleatorizaciones con el estimador Jacknife de primer orden (Colwell 1997).

Se obtuvo la riqueza de especies como la suma del total de especies encontradas en las 20 trampas colocadas por sitio y temporada. Con los datos de abundancia se construyeron curvas de rango-abundancia para conocer la dominancia numérica de las especies. Considerando que la abundancia de los insectos sociales, como las hormigas, puede ser afectada por la cercanía de las trampas a los nidos o a las hileras que estos insectos hacen para forrajear, los datos fueron transformados usando el logaritmo natural (ln) de la abundancia (Bestelmeyer y Wins 2001). Para conocer si la abundancia y riqueza de hormigas de la Cantera Oriente difiere entre sitios y estaciones, se hizo un análisis multivariado de varianza de dos vías por permutaciones (PERMANOVA) y como medida de similitud se usó la distancia de Bray-Curtis. Los factores del análisis fueron el sitio con dos 
niveles (Sendero y Jardín), y la temporada, también con dos niveles (lluvia y sequía). Estas pruebas se hicieron utilizando el programa PAST versión 3.0.

Una vez conocidas la riqueza y la abundancia se calculó el índice de diversidad de Shannon y la equitatividad de Pielou para cada sitio y temporada. Para saber si había diferencias entre los índices de diversidad, se hizo una prueba de t para índices de diversidad con el programa PAST versión 3.0, para disminuir el efecto de las comparaciones pareadas se usó la corrección de Bonferroni (Magurran 1998).

Estructura de la vegetación y de la superficie del suelo. Para conocer la complejidad de la vegetación se midió la estructura vertical de la vegetación utilizando el método de interceptos verticales de la vegetación (Bestelmeyer y Wiens 1996). En los mismos puntos en los que se colocaron las trampas, se utilizó una pértiga de PVC de $3 \mathrm{~m}$ de altura. La pértiga estaba dividida cada $10 \mathrm{~cm}$ para marcar la altura a la que era interceptada por la vegetación. Las alturas se clasificaron en tres categorías para conocer el estrato dominante de cada sitio: herbáceas (plantas menores a $30 \mathrm{~cm}$ ), arbustos (plantas de entre 31 y $150 \mathrm{~cm}$ ) y plantas arbóreas (mayores a $150 \mathrm{~cm}$ ). Se comparó entre sitios la frecuencia de toques de los tres estratos por medio de una tabla de contingencia (Dytham 2011).

Se conoció la complejidad de la superficie del suelo midiendo su estructura, para lo cual se fotografió un área de 20 $\mathrm{cm}^{2}$ junto a cada trampa con una cámara digital (Olympus, modelo VG-120), a $30 \mathrm{~cm}$ de altura. Estas fotografías se capturaron en formato JPG y sobre cada una se dibujó digitalmente una cuadrícula de diez por diez cuadros de $2 \mathrm{~cm}^{2}$ cada uno, con esto se cuantificó la proporción de suelo desnudo, roca, pasto y hojarasca (Ríos-Casanova et al. 2015). Se comparó la proporción de cada categoría entre sitios y estaciones usando un análisis de varianza de una vía con la transformación arcoseno (Zar 1990).

Relación diversidad-parámetros ambientales. Para saber si la estructura de la superficie del suelo (de cada una de las cuatro variables medidas) y la estructura vertical de la vegetación están relacionadas con la abundancia y riqueza de hormigas encontradas, se realizaron correlaciones de Sperman con el programa SPSS versión 3.1.2.

Similitud con la zona núcleo de la reserva. Por último se comparó la riqueza de hormigas de la Cantera Oriente con la riqueza obtenida en estudios previos realizados en la Zona Núcleo Poniente de la Reserva (zona conservada), (Ríos-Casanova et al. 2017) por medio del índice de Sorensen $\mathrm{I}_{\mathrm{s}}=$, donde a es el número de especies de la Cantera Oriente, $\mathrm{b}$ es el número de especies de la Zona Núcleo Poniente y c es el número de especies compartido entre estas dos zonas.

\section{RESULTADOS}

Diversidad de hormigas. Se obtuvieron diez especies de ocho géneros (Cuadro 1). La especie más abundante fue Monomorium minimum, en contraste con Temnothorax nitens de la cual se recolectaron un menor número de obreras
(Cuadro 1).

Las curvas de acumulación de especies indican que se ha colectado entre el $67 \%$ (Jardín en lluvia) y el $90 \%$ (Sendero en lluvia) del total de especies calculadas con el estimador Jacknife de primer orden que es de 11 especies para el sitio Sendero en ambas estaciones y de nueve y diez especies para el sitio Jardín en lluvia y sequía respectivamente. Sin embargo el análisis del comportamiento de las curvas indica que aún se pueden esperar más especies en todos los sitios pues las curvas esperadas no se acercan a la asíntota excepto para el sitio Sendero en la época de sequía (Fig. 1A).

Las curvas de rango abundancia muestran que durante la sequía hay mayor abundancia y que la especie dominante en ambos sitios fue $M$. minimum seguida de P. longicornis (Fig. 1B).

No se encontraron diferencias significativas entre sitios ni entre temporadas para la abundancia (sitio: $\mathrm{F}=1.3, \mathrm{p}=$ 0.3 ; temporada: $\mathrm{F}=0.6, \mathrm{p}=0.7$; interacción sitio por temporada: $\mathrm{F}=0.07, \mathrm{p}=0.9$ ), ni para la riqueza (sitio; $\mathrm{F}=0.3$, $\mathrm{p}=0.8$; temporada: $\mathrm{F}=1.8, \mathrm{p}=0.2$; interacción sitio por temporada: $\mathrm{F}=0.2, \mathrm{p}=0.9$ ).

Los valores de equidad y diversidad tampoco fueron diferentes entre sitios y estaciones. Para la diversidad no se encontraron diferencias significativas $\left(t_{\mathrm{H}}\right.$ todas entre $-0.6 \mathrm{y}$ $0.7 \mathrm{p}>0.05$; Cuadro 1).

Estructura de la vegetación y de la superficie del suelo. La estructura vertical de la vegetación en ambos sitios de la cantera fue escasa, el número de toques para los estratos menores a $30 \mathrm{~cm}$, entre 31 y $150 \mathrm{~cm}$ y mayor a $150 \mathrm{~cm}$ fue de 3, 0 y 10 para el Jardín, y de 1,0 y 5 para el Sendero (Fig. 2A). La estructura de la superficie del suelo mostró una mayor complejidad que la vegetación (Fig. 2B). En ambos sitios, el pasto ocupó la mayor parte de la superficie, seguido de la hojarasca. Las rocas y el suelo desnudo ocuparon una proporción muy pequeña de la superficie. Al analizar si hay diferencias entre estas cuatro categorías entre sitios y estaciones encontramos que no hay efecto del sitio (suelo: $F=0.53, p=0.47$; roca: $F=0.04, p=0.84$; pasto: $F=0.004, p=0.95$; hojarasca: $F=0.03, p=0.85$ ), pero sí de la temporada (roca: $\mathrm{F}=6.6, \mathrm{p}=0.01$; pasto: $\mathrm{F}=$ $10.04, p=0.002$; hojarasca: $F=33.8, p<0.001$ ), excepto para el suelo desnudo donde solo la interacción sitio por temporada fue significativa $(F=7.3, p=0.009$; Fig. 2B).

La mayoría de las correlaciones entre la proporción de suelo desnudo, rocas, pasto, hojarasca o estructura vertical de la vegetación con la abundancia o riqueza de especies de hormigas no fueron significativas. Únicamente, durante la temporada de lluvia, la abundancia se correlacionó con la proporción de pasto (Cuadro 2).

Similitud con la zona núcleo: La riqueza conocida hasta el momento para la Zona Núcleo Poniente de la REPSA es de 28 especies (Ríos-Casanova et al., 2017), de las cuales, siete se comparten con las que se encontraron en la Cantera Oriente, por lo que al calcular el índice de Sorensen se obtuvo un valor de 0.37 . 


\section{DISCUSIÓN}

En este estudio se encontró que por lo menos diez especies de hormigas habitan actualmente en la Cantera Oriente, a pesar de la historia de manejo y restauración que ha tenido este lugar. El factor más importante que actúa sobre la diversidad es la estacionalidad ya que, aunque no hubo diferencias significativas entre sitios ni estaciones, durante la sequía se presentan una mayor riqueza y abundancia de hormigas que en época de lluvia.

En la estación seca se encontró que M. mínimum es una especie numéricamente dominante mientras que en la estación lluviosa $P$. longicornis y D. pyramucus son las especies que dominan. Por lo tanto hay un recambio en las especies, pero no en las abundancias totales por lo que estadísticamente no hay diferencias. A pesar de lo anterior el resto de las especies presentes en la estación seca (como las del género Pheidole) incrementan la abundancia total en la época de sequía. Este patrón no es extraño para comunidades de insectos pues ya se había encontrado en otras comunidades de hormigas (Delssine et al. 2010; Neves et al. 2010) y para otros artrópodos (Andresen 2008; Ríos-Casanova et al. 2010) que durante la sequía los parámetros de diversidad no cambian con respecto a la época lluviosa o inclusive aumentan (Coelho y Ribeiro 2006).

El aumento en la abundancia de hormigas durante la estación seca puede deberse a que, si bien en la temporada de lluvia se dispara la productividad primaria de la reserva ecológica del Pedregal de San Ángel (Cano-Santana 1994), la producción de frutos y semillas, que son utilizadas por muchas hormigas como recurso, se producen al terminar la temporada de lluvia, como ocurre con muchos pastos y asteráceas (César 2002).

Algunos artrópodos, que son recurso para las hormigas omnívoras y depredadoras, también tienen una alta abundancia en la época seca (Ríos-Casanova et al. 2010). Además, durante la sequía el forrajeo puede verse favorecido pues los ambientes con mucha humedad no son adecuados para la locomoción de las hormigas ya que pueden encontrar sitios con mucha agua, o con vegetación muy alta u hojarasca que impida su desplazamiento por la superficie (Lassau y Hochuli 2004). Sin embargo, en este estudio no se encontró que la proporción de hojarasca, de suelo desnudo, de rocas en la superficie del suelo, o la estructura vertical de la vegetación estuvieran relacionadas con la abundancia o riqueza de hormigas de La Cantera Oriente. Otros estudios en los que se ha analizado si existe una relación entre la estructura de la superficie del suelo o de la vegetación (usadas como medidas de la complejidad del hábitat) con la diversidad de hormigas, tampoco han encontrado correlaciones (Ríos-Casanova et al. 2015) o han mostrado correlaciones negativas lo que apoya la idea de que el desplazamiento de estos insectos puede afectarse cuando hay elementos del ambiente que disminuyen la locomoción de las hormigas, lo que se ve reflejado en riquezas y abundancias bajas (Lassau y Hochuli 2004; Delssine et al. 2010).

Finalmente, muchas de las especies de hormigas encon- tradas en La Cantera Oriente tienen hábitos alimentarios generalistas, como P. obtusospinosa, C. atriceps, T. nitens y L. niger, las cuales solo se recolectaron durante la estación seca. En otros ecosistemas, las especies generalistas se ven favorecidas durante esta estación ya que pueden hacer uso de casi de cualquier recurso disponible, pero principalmente porque durante este periodo, puede disminuir la dominancia de algunas hormigas, permitiendo la presencia de aquellas que no pueden competir con las dominantes (Neves et al. 2010).

En el presente estudio, únicamente la proporción de pasto se correlacionó positivamente con la abundancia de hormigas durante la temporada de lluvia y marginalmente durante la época seca. Como se ha encontrado en algunos ecosistemas tropicales, la presencia de pastos favorece a algunas especies de hormigas que consumen los exudados producidos por áfidos que viven en las raíces de estas plantas (Perfecto y Vandermeer 1996). Aunque no se tiene evidencia de este tipo de interacciones en la Cantera Oriente, existe la posibilidad de que esté ocurriendo una interacción similar lo cual deberá ser estudiado en el futuro.

Especies como P. longicornis y M. minimum presentaron abundancias muy altas durante la época seca y la de lluvia. Estas hormigas y algunas del género Pheidole se caracterizan por ser poligínicas y omnívoras (Alder y Silverman 2006). Estas características les permiten tener colonias numerosas y sobrevivir explotando casi cualquier recurso disponible en cualquier época del año, por lo que estas hormigas pueden desarrollarse en sitios donde viven los humanos como son las ciudades o áreas muy urbanizadas, y adaptarse a un amplio rango de condiciones (Thompson y McLachlan 2007). La presencia de estas especies en La Cantera Oriente, así como la baja diversidad encontrada, puede relacionarse con el alto grado de disturbio ocurrido en este sitio y la posterior introducción de plantas exóticas.

Con respecto a la composición total de especies de $\mathrm{La}$ Cantera Oriente, es importante tener en cuenta el poco tiempo que ha transcurrido desde que se hicieron esfuerzos por restaurar este lugar (21 años), ya que se ha visto que el tiempo necesario para que la composición original de la fauna de un sitio restaurado se restablezca puede ser mayor a 37 años aunque es muy probable que la composición de especies de hormigas no llegue a ser como la original (Majer et al. 2013). Por lo tanto es posible que la comunidad de hormigas de la Cantera Oriente no vuelva a ser como lo era antes de haber sido perturbada.

Al comparar las especies de hormigas encontradas en la Cantera Oriente con las especies de la Zona Núcleo Poniente, encontramos que ésta última tiene casi tres veces más especies entre las que se encuentran Nylanderia bruesii,(Wheeler) Formica montana Wheeler y F. subcyanea Wheeler, Solenopsis geminata (Fabricius) y S. bicolor (Emery), así como Neivamyrmex rugulosus Bormeier entre otras (Ríos-Casanova et al. 2017). La Zona Núcleo Poniente es una de las zonas mejor conservadas de la reserva del Pedregal de San Ángel, aunque no ha estado exenta de 
perturbaciones, es un sitio altamente heterogéneo con una topografía muy accidentada, con grietas y sitios planos y con más de 321 especies de plantas (Lot et al. 2007; Lot y Cano-Santana 2009).

Los resultados presentados concuerdan con las hipótesis de que a mayor complejidad del hábitat habría mayor número de especies, sin embargo es necesario hacer más medidas de la complejidad y/o heterogeneidad del hábitat para correlacionarlas con la diversidad de hormigas. En el caso de la REPSA, serán necesarios más estudios que consideren la variación temporal en la heterogeneidad del ambiente, así como el uso de otros métodos de colecta como los extractores Winkler, diferentes tipos de cebos y redes de golpeo en la vegetación que permitan colectar hormigas asociadas a otros estratos diferentes al suelo que fue el objetivo del presente estudio (Bestelmeyer y Ríos-Casanova 2010).

Otros estudios con comunidades de hormigas han encontrado que efectivamente hay una relación entre la heterogeneidad y la diversidad de hormigas (Perfecto y Snelling 1995; Retana y Cerdá 2000; Pacheco y Vasconcelos 2012) aunque para otras comunidades esta relación ha sido negativa (Lassau y Hochuli 2004; Coelho y Ribeiro, 2006; Travassos-Brito y Rocha 2018).

Los resultados de este trabajo permiten concluir que, a pesar de la historia de disturbio y posterior restauración que ha tenido la Cantera Oriente de la REPSA, al menos diez especies de hormigas pueden vivir en este lugar y que la variación estacional es el factor que tiene más efecto sobre su diversidad. Con respecto a la relación que puede haber entre la complejidad del ambiente y la diversidad, encontramos que las zonas más conservadas de la REPSA (las zonas núcleo) que son más complejas, tienen hasta tres veces más especies de hormigas, lo que podría estar apoyando esta hipótesis. Será necesario hacer otros estudios donde se mida explícitamente la heterogeneidad o complejidad del ambiente, con especial énfasis en los cambios estacionales de la vegetación.

\section{AGRADECIMIENTOS}

Este estudio se realizó con el apoyo de la Universidad Nacional Autónoma de México-Dirección General de Asuntos del Personal Académico-Programa de Apoyo a Proyectos de Investigación e Innovación Tecnológica IN 216714. Agradecemos al comité técnico de la Reserva Ecológica del Pedregal de San Ángel (2014-2018) y a Francisco Martínez Pérez por otorgarnos los permisos para trabajar en la Cantera Oriente. Finalmente a Miguel Vásquez Bolaños por ayudarnos con la identificación de las hormigas.

\section{LITERATURA CITADA}

Alder, A. and J. Silverman. 2006. Effects of interspecific competition between two urban ant species, Linepithema humile and Monomorium minimum, on toxic bait performance. Journal of Economic Entomology, 98 (2): 493-501.

Álvarez-Sánchez, F.J., J. Carabias, J. Meave, P. Moreno-Ca- sasola, D. Nava-Fernández, D. Rodriguez-Zahar, F.C. Tovar y A. Valiente-Banuet. 1982. Proyecto para la creación de una reserva en el Pedregal San Ángel. Serie de Cuadernos de Ecología No.1. Facultad de Ciencias, UNAM. D. F.

Andresen, E. 2008. Dungle beetle assemblages in primary forest and disturbed habitats in a tropical dry forest landscape in western Mexico. Journal Insect Conservation, 12 (6): 639-650.

Bestelmeyer, T. B. and J. A. Wiens. 1996. The effects of land use on the structure of ground-foraging ant communities in the Argentine Chaco. Ecological Applications, 6 (4): 1225-1240.

Bestelmeyer, B. T. and J. A. Wiens. 2001. Ant biodiversity in semiarid landscape mosaics: the consequences of grazing vs. natural heterogeneity. Ecological Applications, 11 (4): 1123-1140.

Bestelmeyer, B. T. \& L. Ríos-Casanova. 2010. Field techniques for sampling ants. (pp. 39-41). In: Lach, L., C. L. Parr \& K. L. Abbott (Eds.). Ant ecology. Oxford University Press, Oxford.

Cano-Santana, Z. 1994. Flujo de energía a través de Sphenarium purpurascens (Orthoptera: Acrididae) y productividad primaria neta aérea en una comunidad xerófita. Tesis doctoral, Universidad Nacional Autónoma de México, México, D.F.

César, G. S. F. 2002. Análisis de algunos factores que afectan la fenología reproductiva de la comunidad vegetal de la Reserva del Pedregal de San Ángel, D.F. (México). Tesis de Licenciatura en Biología. Universidad Nacional Autónoma de México, México, D.F.

Chávez, C. y G. Ceballos. 1998. Diversidad y estado de conservación de los mamíferos del Estado de México. Revista Mexicana de Mastozoología 3:113-134.

Clarke, M. K., B. L. Fisher and G. LeBuhn. 2008. The influence of urban parks characteristics on ant (Hymenoptera, Formicidae) communities. Urban Ecosystems, 11 (3): $317-334$.

Coelho, I. R. and S. P. Ribeiro. 2006. Environmental heterogeneity and seasonal effects in ground-dwelling ant (Hymenoptera: Formicidae) assemblages in the Parque Estadual do Rio Doce, MG, Brazil. Neotropical Entomology, 35 (1): 19-29.

Colwell, R. K. 1997. EstimateS: Statistical estimation of species richness and shared species from samples, Version 5, User's Guide and application, Recuperado de: http://viceroy.eeb.unconn.edu/estimates.

Delssine, T., Y. Roisin, J. Herbauts and M. Leponce. 2010. Ant diversity along a wide rainfall gradient in the Paraguayan dry Chaco. Journal of Arid Environments, 74 (10): 1149-1155.

Dytham, C. 2011. Choosing and using statistics. A biologist's guide. Oxford Blakwell Piblishing. London.

Fisher, B. L. and S. P. Cover. 2007. Ants of North America: a guide to the genera. University of California Press. London. 
Folgarait, J. P. 1998. Ant biodiversity and its relationship to ecosystem functioning: a review. Biodiversity and Conservation, 7 (9): 1221-1224.

Hölldobler, B. and E. O. Wilson. 1990. The Ants. Belknap Harvard University Press. Cambridge.

Lassau, S.A and D. F. Hochuli. 2004. Effects of habitat complexity on ant assemblages: can we generalise across scale? Ecography, 27 (2): 157-164.

Lot, A. 2007. Guía ilustrada de la Cantera Oriente. Caracterización ambiental e inventario biológico. Universidad Nacional Autónoma de México, México.

Lot, A. y Z. Cano-Santana. 2009. Biodiversidad del ecosistema del Pedregal de San Ángel. Universidad Nacional Autónoma de México, México, D. F.

Lot, A., M. Pérez-Ecobedo, G. Gil-Alarcón, S. Rodríguez-Palacios y P. Camarena. 2012. La reserva ecológica del Pedregal de San Ángel: Atlas de riesgos. UNAM, ICyTDF, Reserva Ecológica del Pedregal de San Ángel., México, D.F.

Magurran, A. E. 1998. Ecological diversity and its measurement. Princeton University Press, Princeton.

Majer, J. D., B. Heterick, T. Ghor, E. Hughes, L. Mounsher and A. Grigg. 2013. Is thirty-seven years sufficient for full return of the ant biota following restoration? Ecological Processes, 2: 19.

Neves, F.S., R.F. Braga, M. M. do Espirito-Santo, J. H. C. Delabie, G. W. Fernandes and G. A. Sanchez-Azofeifa. 2010. Diversity of arboreal ants in a Brazilian tropical dry forest: Effects of seasonality and successional stage. Sociobiology, 56 (1): 1-18.

Pacheco, R. and H. L. Vasconcelos. 2007. Invertebrate conservation in urban areas: ants in the Brazilian Cerrrado. Landscape Urban Planning, 81: 193-199.

Pacheco, R. and H. L. Vasconcelos. 2012. Habitat diversity enhances ant diversity in a naturally heterogeneous Brazilian landscape Biodiversity and Conservation, 21 (3): 797-809.

Recibido: 6 de agosto 2019

Aceptado: 2 de diciembre 2019
Perfecto, I., and R. Snelling. 1995. Biodiversity and the transformation of a tropical agroecosystem: ants in coffee plantations. Ecological Applications, 5 (4): 1084 1097.

Perfecto, I. and J. Vandermeer. 1996. Microclimatic changes and the indirect loss of ant diversity in a tropical agroecosystem. Oecologia, 108 (3): 577-582.

Retana, J. and X. Cerdá. 2000. Patterns of diversity and composition of Mediterranean ground ant communities tracking spatial and temporal variability in the thermal environment. Oecología, 123 (3): 436-444.

Ríos-Casanova, L., Z. Cano-Santana and H. Godínez-Alvarez. 2010. Patterns of arthropod diversity in contrasting habitats of El Pedregal de San Angel, a preserve in Mexico City. Southwestern Entomologist, 35 (2): 165-175.

Ríos-Casanova, L., P. Davila, H. Godínez-Alvarez, and V. Rico-Gray. 2015. Diversity of ants inhabiting a mosaic of environmental conditions in a Semi-Desert of central Mexico. Southwestern Entomologist, 40 (2): 307-322.

Ríos-Casanova, L., K. Baltazar, Z. Cano-Santana, L. González, P. García, C. Jímenez, R. Trejo, J. Valentín and M. Vásquez-Bolaños. 2017. Species richness of ants of the Pedregal de San Ángel, an ecological reserve in Mexico in City. Southwestern Entomologist, 42 (3): 923- 926.

Santos, M.N. 2016. Research on urban ants: approaches and gaps. Insectes Sociaux, 63 (3): 359-371.

Tews, J., U. Brose, V. Grimm, K. Tielbörger, M. C. Wichmann, M. Schwager, and F. Jeltsch. 2004. Animal species diversity driven by habitat heterogeneity/diversity: the importance of keystone structures. Journal of Biogeography, 31 (1): 79-92.

Thompson, B. and S. McLachlan. 2007. The effects of urbanization on ant communities and myrmecochory in Manitoba, Canada. Urban Ecosystems, 10 (1): 43-52.

Travassos-Britto, B. and P. B. L. Rocha. 2018. Habitat heterogeneity reduces richness of ant species by increasing abundance of the local dominant species. bioRxiv http:// dx.doi.org/10.1101/316430

Zar, J.H. 1990. Statistical analysis. Prentice Hall, New Jersey. 
1

A

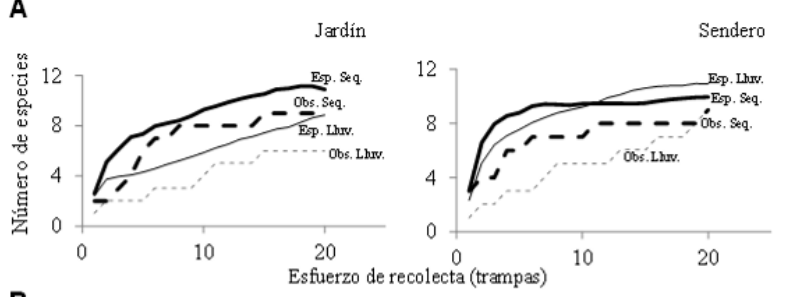

B

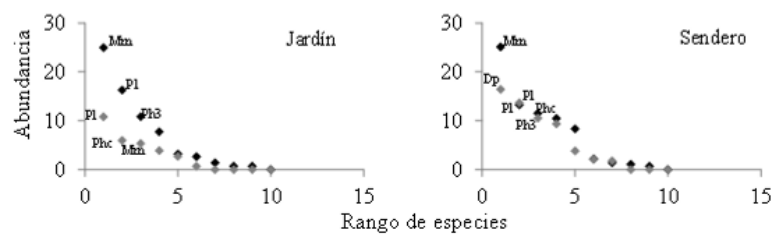

2

A

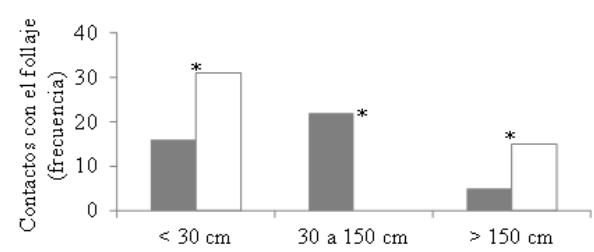

B

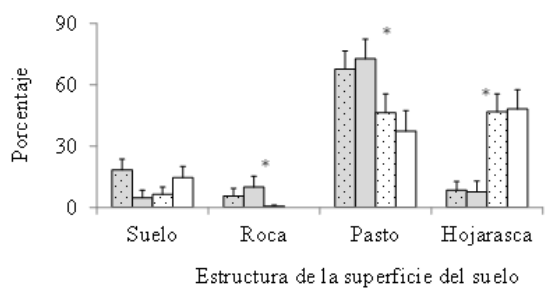

Fig. 1. Curvas de acumulación de especies (A) y de rango-abundancia (B) para las hormigas de la Cantera Oriente de la Reserva Ecológica Pedregal de San Ángel. (A) especies observadas (líneas punteada) y esperadas (línea continua) para dos sitios en época de lluvia (líneas delgadas) y sequía (líneas gruesas). (B) época de sequía (negro) y de lluvia (gris). $\mathrm{Mm}=$ Monomorium mínimum, $\mathrm{Pl}=$ Paratrechina longicornis, $\mathrm{Phc}=$ Pheidole coracina, $\mathrm{Ph} 3=$ Pheidole $\mathrm{sp} .3, \mathrm{Dp}=$ Dorymyrmex pyramicus .

Fig. 2. Estructura vertical de la vegetación y de la superficie del suelo. A) Número total de contactos con el follaje en jardín (gris) y sendero (blanco) $*=$ diferencias significativas $\left(\mathrm{X}^{2} \mathrm{p}<0.05\right)$. B) porcentaje promedio de las categorías en que se dividió la estructura de la superficie del suelo en lluvia (gris) y sequía (blanco) en el sendero (punteado) y el jardín (liso). * diferencias significativas ANOVA $\mathrm{p}<0.05$.

Cuadro 1. Riqueza, abundancia (ln), diversidad y equidad de las comunidades de hormigas en el jardín y el sendero de La Cantera Oriente del Pedregal de San Ángel, en dos estaciones.

\begin{tabular}{|c|c|c|c|c|}
\hline \multirow{2}{*}{$\begin{array}{c}\text { Subfamilia } \\
\text { Especie }\end{array}$} & \multicolumn{2}{|l|}{ Jardín } & \multicolumn{2}{|l|}{ Sendero } \\
\hline & Sequía & Lluvia & Sequía & Lluvia \\
\hline \multicolumn{5}{|l|}{ Dolichoderinae } \\
\hline Dorymyrmex pyramicus (Roger, 1863) & 2.6 & 3.9 & 10.4 & 16.4 \\
\hline \multicolumn{5}{|l|}{ Formicinae } \\
\hline Camponotus atriceps (Smith, 1858) & 0.7 & 0 & 0 & 0 \\
\hline Lasius niger (Linnaeus, 1758) & 0.7 & 0 & 0.7 & 1.8 \\
\hline Paratrechina longicornis (Latreille, 1802) & 16.3 & 10.8 & 13.3 & 13.7 \\
\hline \multicolumn{5}{|l|}{ Myrmicinae } \\
\hline Monomorium minimum (Buckley, 1866) & 24.9 & 5.3 & 25.1 & 3.8 \\
\hline Pheidole coracina Wilson, 2003 & 7.7 & 5.9 & 11.5 & 9.3 \\
\hline Pheidole obtusospinosa Pergande, 1896 & 3.2 & 0 & 2.2 & 0 \\
\hline Pheidole sp.3 & 10.8 & 2.7 & 8.3 & 10.5 \\
\hline Solenopsis stricta Emery, 1896 & 1.4 & 0.7 & 1.4 & 2.1 \\
\hline Temnotorax nitens (Emery, 1895) & 0 & 0 & 1.1 & 0 \\
\hline Riqueza & 9 & 6 & 9 & 7 \\
\hline Abundancia $(\ln )$ & 68.4 & 29.4 & 74.0 & 57.6 \\
\hline Diversidad (Shannon-Wiener) & 1.7 & 1.6 & 1.8 & 1.71 \\
\hline Equidad (Pielou) & 0.77 & 0.88 & 0.81 & 0.88 \\
\hline
\end{tabular}


Cuadro 2. Coeficientes de correlación de Sperman entre variables ambientales (estructura de la superficie del suelo y estructura vertical de la vegetación) y la abundancia o riqueza de hormigas de la Cantera Oriente del Pedregal de San Ángel.

\begin{tabular}{lllllllll}
\hline & \multicolumn{2}{l}{\begin{tabular}{l} 
Abundancia \\
\multicolumn{2}{l}{ Sequía }
\end{tabular}} & \multicolumn{3}{c}{ Riqueza } \\
& $r_{s}$ & $P$ & $r_{s}$ & $p$ & $r_{s}$ & $p$ & $r_{s}$ & $P$ \\
\hline Suelo desnudo & 0.16 & 0.3 & -0.14 & 0.4 & 0.17 & 0.3 & -0.13 & 0.4 \\
Roca & -0.02 & 0.9 & -0.06 & 0.7 & 0.03 & 0.9 & 0.11 & 0.5 \\
Pasto & -0.06 & 0.7 & 0.3 & 0.04 & -0.05 & 0.8 & 0.3 & 0.08 \\
Hojarasca & 0.1 & 0.5 & -0.2 & 0.2 & -0.08 & 0.6 & -0.16 & 0.3 \\
Vegetación & - & - & -0.15 & 0.5 & - & - & -0.33 & 0.2 \\
\hline
\end{tabular}

